\title{
The predictive value of a targeted posterior fossa multimodal stroke protocol for the diagnosis of acute posterior ischemic stroke
}

\author{
Michal Sharon ${ }^{1}$, Karl Boyle², Robert Yeung ${ }^{1}$, Liying zhang ${ }^{1}$, Sean P. Symons', Mark I. Boulos ${ }^{2}$ and Richard I. Aviv ${ }^{\text {1* }}$
}

\begin{abstract}
Background: There is limited but growing research regarding the accuracy of CTP in diagnosing acute posterior ischemia stroke. We sought to evaluate the diagnostic accuracy of an incremental multimodal CT protocol in acute posterior ischemic stroke.

Methods: Retrospective review of incremental NCCT, CTA-source images and CTP use in 82 consecutive patients with acute posterior ischemic stroke. Readers were blinded to infarct status on follow-up imaging (MRI or CT). Predictive effects of observed diagnostic accuracy and confidence score were quantified with the entropy $r^{2}$ value. Sensitivity, specificity, and Cl were calculated accounting for multiple reader assessments. Receiver Operating Characteristic analyses, including Area Under the Curve, were conducted for the three modalities. Inter-reader agreement was established with Intraclass Correlation Coefficient.

Results: Follow-up imaging confirmed infarct in 69/82 (84 \%) patients. Multimodal protocol with CTP, outperforms CTA-source images and NCCT for correct acute posterior ischemia stroke diagnosis. The Area Under the Curve was 0.741 ( $95 \% \mathrm{Cl} 0.708-0.773$ ); 0.70 (95 \% Cl 0.663-0.731, $P=0.03$ ) and 0.62 (95\% Cl 0.588-0.659, $P<0.0001$ ), respectively. Incrementally improved correlation between observed and actual diagnosis $\left(r^{2}=0.09,0.26\right.$ and 0.32$)$ and a higher rate of certainty (51.4, 69.3 and $81.7 \%$ ) was demonstrated for NCCT, CTA-source images and CTP respectively. Inter-reader agreement for the actual diagnosis was good and improved from 0.68 to 0.83 with incremental multimodal CT use.
\end{abstract}

Conclusions: CTP enhances confident and correct infarct diagnosis over NCCT and CTA-source images in acute posterior ischemia stroke.

Keywords: Posterior fossa stroke, Ischemia, Infarct, CTP, Acute ischemic stroke, Multimodal CT, Posterior circulation

\section{Background}

Acute posterior ischemia stroke accounts for $20 \%$ of ischemic stroke [1] and is most commonly cardioembolic followed by large-artery atherosclerosis [2]. Non contrast CT (NCCT) performs poorly at detecting acute posterior-fossa stroke even utilizing posterior circulation Alberta stroke program early CT score (ASPECTS) [3], largely due to beam hardening artifacts and insufficient contrast resolution. MRI and particularly DWI remains the mainstay of infarct diagnosis but is not yet widely adopted in acute stroke imaging due to its

\footnotetext{
* Correspondence: richard.aviv@sunnybrook.ca

'Department of Diagnostic Imaging, Division of Neuroradiology, Room AG 31, Sunnybrook Health Sciences Centre, 2075 Bayview Ave, Toronto, ON M4N 3M5, Canada

Full list of author information is available at the end of the article
}

reduced availability, increased cost and scan time. Although the reference standard, DWI demonstrates reduced sensitivity in the context of small and posterior fossa infarction [4]. Both CT angiography (CTA) and $\mathrm{CT}$ perfusion (CTP) are previously evaluated in the posterior fossa. CTA-source image (CTA-SI) hypoattenuation is shown to improve detection of posterior fossa infarction and is predictive of clinical outcome [5]. CTP is fast and effective in the emergency evaluation of acute anterior-circulation infarction [6-8] and increases diagnostic certainty for clinical stroke by expert and nonexpert readers over CTA-SI [7] and correlates with MRI DWI [9]. Drawbacks of CT include radiation dose and limited spatial coverage with many centres omitting the posterior-fossa by necessity in favour of supratentorial 
coverage. CTP however can be directed to evaluate the posterior fossa according to clinical presentation. Literature regarding the diagnostic performance of posteriorfossa CTP is limited but growing with only three recently published articles [10-12]. Advances in CT such as a table-toggle technique or increase detector number [13], now facilitate more extensive brain coverage with increasing visualization of the posterior fossa. However this extended coverage is not yet widely available. With increasing CTP imaging of the posterior fossa expected, further study of the strengths and limitations of CTP within the context of posterior ischemic stroke is needed. The purpose of this study was therefore to assess the predictive value of each component (NCCT, CTA-SI and CTP) of an incremental CT protocol using targeted posterior fossa CTP for the assessment of posterior ischemic stroke presentation.

\section{Methods}

\section{Ethics, consent and permissions}

The study was approved by the local institutional research ethics board (Research Ethics Human Research Program, Sunnybrook Health Sciences Centre).

Signed was obtained from each patient or substitute decision maker for study enrollment and publication.

\section{Study design and patients}

A retrospective cohort of consecutive acute posterior ischemia stroke patients presenting to a tertiary strokecenter emergency department between 2010 and 2012 . Targeted posterior fossa CTP was performed prospectively in patients presenting with signs and symptoms of acute posterior ischemia stroke, diagnosed by an experienced Neurologist (xx, 7 years) within $12 \mathrm{~h}$ of symptom onset. Patients were included if their final diagnosis based on clinical and imaging findings was TIA or infarct as arbitrated by the Neurologist. Patients with stroke mimics, fetal posterior cerebral artery on baseline CTA or those with contraindications to iodinated-contrast such as allergy or severe renal impairment $(G F R<20)$ were excluded. Patients with intracranial stenosis were not excluded from the cohort. Baseline clinical data included gender, age, cardiovascular risk factors, NIHSS (National Institutes of Health Stroke Scale) score, rtPA status and dose.

\section{CT protocol}

The CT stroke protocol was performed on a 64-section VCT (GE Healthcare). Aortic-arch to vertex CTA was obtained [7]. CTA source-image (CTA-SI) reformations (4 mm thick, 2-mm gap) were aligned to match the NCCT image. The 40-mm slab biphasic CTP commenced inferior to the frontal horns covering most of the posterior fossa, yet retaining visualization of the bilateral anterior cerebral artery and torcula for ROI placement. First phase was $45 \mathrm{~s}$ scan reconstructed at $0.5 \mathrm{~s}$ intervals followed by 6 further acquisitions $15 \mathrm{~s}$ apart for an additional 90s. CTP parameters are: 80 $\mathrm{kVp}, 100 \mathrm{~mA}, 0.5 \mathrm{ml} / \mathrm{kg}$ (maximum $50 \mathrm{ml}$ ) iodinated contrast agent injected at $4 \mathrm{ml} / \mathrm{s}$ with a 3-5 s delay [7]. Effective-doses of individual components are NCCT $1.2 \mathrm{mSV}, \mathrm{CTA} 2.4 \mathrm{mSV}$ and CTP $2.5 \mathrm{mSV}$. Lifetime attributable risk of cancer for NCCT at the median age of the studied cohort is $0.01 \%$ [14]. Follow-up was performed at 5-7 days on MRI (57/82; $70 \%$ ), unless a readily visible hypodensity involving the affected vascular territory was demonstrated on follow-up NCCT (25/82;30 \%). MRI minimally included DWI/ADC maps $(\mathrm{TR}=8125 \mathrm{~ms} / \mathrm{min} \mathrm{TE} ; 26-\mathrm{cm}$ field of view; $128 \times 128$ matrix; 5-mm section thickness; no intersection gap) and FLAIR $(\mathrm{TR}=8000 \mathrm{~ms} / \mathrm{TE}=120 \mathrm{~ms} / \mathrm{TI}=200 \mathrm{~ms}$; 22 -cm field of view; $320 \times 224$ matrix; 5 -mm section thickness; 1-mm intersection gap).

\section{Image processing}

Commercially available software (CTP4; GE Healthcare) was used to calculate CTP maps with colorcoding of cerebral blood volume (CBV), cerebral blood flow (CBF) and mean transit time (MTT). Generation of arterial and venous time attenuation curves was based on manual selection of the arterial input function (AIF) within the anterior cerebral artery and the torcula, respectively [7].

\section{Review protocol}

Images were reviewed by three readers blinded to imaging outcome; including a neuroradiologist (4 years of experience), a neuroradiology fellow and a neurology fellow (2 years' experience each). The review method simulated the clinical review process, beginning with NCCT, followed by CTA-SI and CTP color maps [7, 15]. Therefore, three DICOM folders were prepared containing NCCT, NCCT + CTA-SI and NCCT + CTA-SI + CTP. In all cases the posterior-circulation ASPECTS [16] regions were specifically reviewed to ensure a systematic approach to scan review and to maximize detection. Images were reviewed independently by the readers in three stages, one for each modality, 2 weeks apart to avoid recall-bias. The readers documented in each step the presence or absence of ischemia/infarction, based on parenchymal hypoattenuation on NCCT (infarct) and CTA-SI (ischemia/infarction) [17] , qualitative MTT prolongation and CTP CBF/CBV reduction. We specifically included qualitative CTP because most centers cannot use thresholded parameters in the acute setting. We justified the inclusion of purely ischemic patterns (MTT prolongation/ $\mathrm{CBF}$ reduction in the absence of a visible CBV defect) in addition to overt infarction (CBV reduction) because even in the context of TIA (with clinical 
improvement), a perfusion abnormality (signifying ischemia) is associated with an increased probability of DWI/ ADC infarction at presentation or follow-up [18, 19]. This approach has been used before in the supratentorium [7] in a recent study of lacunar infarction [15] and posterior fossa CTP [12]. For each posterior fossa ASPECTS location a positive diagnosis required localization to right, left or midline and was scored individually for each modality (total scores 738 for 3 readers). Therefore a positive diagnosis in an incorrect location was considered a false-positive for that location and a false-negative observation for the true infarct location. A six-point level of confidence-score was assigned (1-infarct/ischemia definitely present; 2 - probably present; 3 -equivocal but suspicious for infarct/ischemia; 4-equivocal but infarct/ischemia presumed absent; 5- infarct/ischemia probably absent; 6- definitely absent). Additionally, readers indicated the affected vascular territory (basilar/perforator, posterior cerebral artery, PICA, AICA, superior cerebellar artery) based on standard vascular anatomy texts. Readers documented their observations separately for each sequence group, without access to prior entries. Final infarct status/ clinical diagnosis was based on DWI/ADC restriction, NCCT hypoattenuation and final clinical diagnosis as assessed by an experienced Neurologist, XX 4 years.

\section{Statistical analysis}

Results were expressed as mean \pm standard deviation or median + interquartile range (IQR) for quantitative variables and as proportions for categorical findings. Logistic-regression analysis predicted actual from observed stroke diagnosis for the incremental protocol adjusting for confidence scores. OR and $95 \% \mathrm{CI}$ were calculated. Actual and observed diagnostic performance was recorded as 0 (absence) and 1 (presence) with 1 level of confidence score 1 to 6 . Combined predictive effects of the observed diagnostic performance and confidence scores in the model were quantified with the entropy $r^{2}$ value, where the higher the $r^{2}$ value the better the model. Akaike information criterion (AIC) was compared among the three incremental review steps with a lower AIC signifying a better model. Diagnostic accuracy of the incremental protocol was evaluated with Receiver Operating Characteristic (ROC) curve and Area under the Curve (AUC) comparisons [20]. Generalized estimating equations calculated real stroke confirmation from observed diagnosis adjusting for confidence score. A generalized linear model with a binomial distribution was performed using the generalized estimating equation method. Quasilikelihood-information criterion (QIC) was applied to the model fit of the generalized estimating equation with smaller values indicating a better model fit. Because of the correlation resulting from multiple reader assessments on the same images and the various CT protocols applying to the same subject, the individual sensitivity $\left(\mathrm{Se}_{\mathrm{i}}\right)$ and specificity $\left(\mathrm{Sp}_{\mathrm{i}}\right)$ were calculated from the cross table of real stroke diagnosis and observed diagnosis for each sequence [21]. Inter-reader agreement was established with intraclass-correlation coefficient (ICC) [22] to estimate inter-reader concordance for the actual diagnosis. ICC of $\leq 0.20,0.21-0.40,0.41-$ $0.60,0.61-0.80$ and $0.81-1.0$ were defined as a poor, fair, moderate, good and very good concordance. A $T$-test was applied to compare ICC generated using the bootstrap method of 1000 generated and calculated sample sets. Analyses were performed with statistical software package (SAS version 9.3; SAS Institute, Cary, NC). Results were considered significant at $p<0.05$.

\section{Results}

There were 82 patients (43/82; $52 \%$ female) with a mean age of $70 \pm 16$ years. Baseline demographic characteristics are listed in Table 1. Infarct was confirmed on follow up imaging in $69 / 82(84 \%)$ of patients, while the remaining 13 patients were diagnosed with TIA; 35/69 (51\%) were female. Median posterior-circulation ASPECTS was 10 (IQR range 9-10) with 149/5, 904 (2.5\%) positive regions (82 patients $\times 3$ readers $\times 8$ regions [16]). Median NIHSS was 4 (IQR 3-7). No statistically significant difference in age, gender, NIHSS or cardiovascular risk factors was present in patients with confirmed infarct versus patients

Table 1 Baseline Demographic Characteristics of 82 patients with suspected acute posterior ischemic stroke

\begin{tabular}{ll}
\hline Female Gender & $43(52)$ \\
Cardiovascular risk factors & \\
Hypertension & $28(34)$ \\
Diabetes mellitus & $33(40)$ \\
Coronary artery disease & $18(22)$ \\
Atrial fibrillation & $37(45)$ \\
Smoking & $34(41)$ \\
Hypercholesterolemia & $15(18)$ \\
Presence of infarct on follow up imaging & $69(84)$ \\
Large vessel infarct* & $63(91)$ \\
Affected side on follow up imaging & \\
Right & $31(45)$ \\
Left & $25(36)$ \\
Midline & $5(7)$ \\
Bilateral & $8(12)$ \\
Imaging follow up & \\
MRl & $57(70)$ \\
CT & $25(30)$ \\
\hline Alvaly &
\end{tabular}

All values represent $n(\%)$

* of 69 patients with confirmed infarct on follow up imaging 
without infarct. Median time from symptom-onset to baseline CT was 115 min (IQR 89-215). Median time to follow-up was 1 day (IQR 0-1). IV rtPA was administered in 23/82 (28\%) with mean dose of $56 \pm 21 \mathrm{mg}$ and within a median of $144 \mathrm{~min}$ (IQR 134-186). No patients received intra-arterial thrombolysis. Twenty (87 \%) of the rtPAtreated patients had confirmed infarct on follow-up imaging. The vascular distribution of posterior ischemic stroke using the incremental CT protocol is summarized on Table 2.

NCCT, CTA and CTP abnormality was present in 43/82 (52.4\%), $44(53.7 \%)$ and 62 (75.6\%) patients and $56(13.7 \%), 93(22.7 \%)$ and 117 (29\%) of 410 vascular territories ( 5 vascular territories $\times 82$ patients) on NCCT, CTA and CTP respectively. The AUC was greater for CTP 0.741 (95\% CI 0.708 - 0.773) compared to CTA-SI 0.70 (95\% CI 0.663-0.731, $P=0.03$ ) and NCCT 0.62 (95\% CI $0.588-0.659, P<0.0001)$, respectively (Fig. 1). Similarly, CTA-SI outperformed NCCT $(P=0.0001)$. For confident diagnosis of infarct absence (Table 3) CTP sensitivity trended $12.6 \%$ higher than CTA-SI $(p=0.072)$ and was significantly higher than NCCT. For confident diagnosis of infarct presence, CTP sensitivity was 15.8 and $30.2 \%$ greater than CTASI and NCCT respectively $(p=0.025$ and $p<0.0001)$ (Figs. 2 and 3). No specificity differences were seen for CTP over CTA-SI although both CTA-SI and CTP showed improvement over NCCT. Table 4 depicts the distribution of the readers' confidence scores for each modality. Significant reduction of equivocal diagnoses is demonstrated between NCCT $(68 ; 9.2 \%)$ and CTASI/ CTP (30; $4.1 \%$ for each modality). More confident diagnosis or infarct exclusion was seen with multimodal CT use (NCCT $51.4 \%$, CTA $69.3 \%$ and CTP $81.7 \%$ ) with higher entropy $r^{2}$ and lower AIC, confirming better correlation between the observed and actual diagnosis.

Progressive entropy $\mathrm{r}^{2}$ increase occurred from 0.09 (NCCT), to 0.26 (CTA-SI) and 0.32 (CTP; Table 5). Similarly, the QIC declined from NCCT to CTA-SI and CTP indicating increasingly better fit between the readers' observed diagnosis and confirmed infarcts. Inter-reader agreement for actual diagnosis was good and improved incrementally with multimodal CT use from 0.59 to 0.87 (Table 6). CTP inter-reader agreement was significantly improved over both NCCT and CTA-SI.

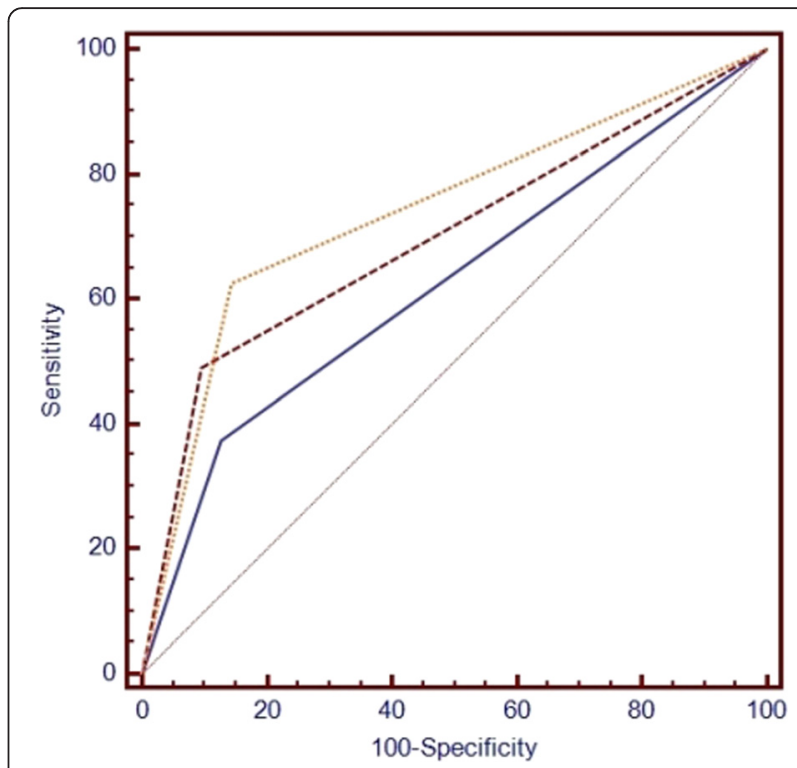

Fig. 1 Receiver Operating Characteristic curves for diagnostic performance of incremental CT protocol for posterior ischemic stroke. The area under the curve of protocol containing targeted posterior fossa CTP (dotted orange line) was greater compared to CTA-SI (dashed brown line, $p=0.03$ ) or NCCT (solid blue line $p<0.0001$ )

\section{Discussion}

A multimodal CT protocol including targeted posteriorfossa CTP improved acute posterior ischemic stroke diagnosis over NCCT alone or a combination of NCCT/ CTA-SI supporting the addition of CTP to stroke protocols using only NCCT and CTA-SI. This assertion is supported by a recent study showing similar improvement for a multimodal approach in the posterior fossa [11]. Extending on these findings, we demonstrate that multimodal CT demonstrated a greater reader certainty for correct diagnosis, compared to CTA-SI or NCCT with a better inter-reader agreement. Correlation between the observed and actual diagnosis, after adjusting for confidence levels, was 3.75 times greater for CTP than NCCT. Sensitivity of the multimodal protocol for correct diagnosis was increased without significant loss of specificity. The sensitivity for NCCT and CTA-SI is similar to that recently reported in the posterior fossa of $31 \%$ and $33 \%$ but lower on CTP (74\%) while specificity was similar for all modailities (93-98\%). Sensitivity of

Table 2 Vascular distribution of infarct/ischemia at presentation on incremental CT protocol

\begin{tabular}{lllllll}
\hline Vascular territory & Basilar & PCA & PICA & AICA & SCA & Perforator/other \\
\hline NCCT & $0(0)$ & $10(0.48)$ & $3(0.14)$ & $1(0.05)$ & $3(0.14)$ & $4(0.19)$ \\
NCCT + CTA-SI & $1(0.17)$ & $2(0.33)$ & $0(0)$ & $0(0)$ & $2(0.33)$ & $1(0.17)$ \\
NCCT + CTA-SI + CTP & $17(0.18)$ & $39(0.42)$ & $10(0.11)$ & $4(0.04)$ & $12(0.13)$ & $11(0.12)$ \\
\hline
\end{tabular}

All values represent $n(\%)$

PCA posterior cerebral artery, SCA superior cerebellar artery, CTA-SI CTA-source images 
Table 3 Diagnostic performance for acute posterior stroke absence or presence diagnosed with an incremental stroke protocol

\begin{tabular}{|c|c|c|c|c|}
\hline & \multicolumn{2}{|l|}{ Sensitivity $(\mathrm{Se})$} & \multicolumn{2}{|l|}{ Specificity $(S p)$} \\
\hline & Se \% $(95 \% \mathrm{Cl})$ & $p$-value* & Sp \% $(95 \% \mathrm{Cl})$ & $p$-value ${ }^{*}$ \\
\hline \multicolumn{5}{|c|}{ Ischemia/ Infarct absent (Level of confidence score 5 and 6) } \\
\hline (1) NCCT & $41.4(32.9-50.0)$ & (1) vs. (2): 0.103 & $83.7(80.0-87.4)$ & (1) vs. (2): $<0.0001$ \\
\hline (2) NCCT + CTA-SI & $52.3(43.3-61.2)$ & (1) vs. (3): 0.0007 & $95.7(93.8-97.6)$ & (1) vs. (3): $<0.0001$ \\
\hline (3) NCCT+ CTA-SI + CTP & $64.9(55.7-74.0)$ & (2) vs. (3): 0.072 & $92.8(90.4-95.2)$ & (2) vs. (3): 0.188 \\
\hline \multicolumn{5}{|c|}{ Infarct present (Level of confidence score $\leq 2$ vs. > 2) } \\
\hline (1) NCCT & $28.8(20.6-37.1)$ & (1) vs. (2): 0.032 & $91.5(88.5-94.4)$ & (1) vs. (2): $<0.0001$ \\
\hline (2) $N C C T+C T A-S I$ & $43.2(33.9-52.6)$ & (1) vs. (3): $<0.0001$ & $97.7(96.4-98.9)$ & (1) vs. (3): 0.002 \\
\hline (3) $\mathrm{NCCT}+\mathrm{CTA}-\mathrm{SI}+\mathrm{CTP}$ & $59.0(49.3-68.7)$ & (2) vs. (3): 0.025 & $96.1(94.2-98.0)$ & (2) vs. (3): 0.318 \\
\hline
\end{tabular}

NCCT non-contrast CT, CTA-SI CTA Source Images, CTP CT perfusion

${ }^{*} p$-value was obtained by linear regression model of natural $\log (\mathrm{Se}) \operatorname{or} \log (\mathrm{Sp})$ for each modality

NCCT in posterior ischemic stroke was expectedly lower than that reported in acute anterior ischemic stroke (28\% vs $52 \%$ ) with closer approximation of CTA-SI ( $43 \%$ vs $58 \%$ ) and CTP (59\% vs $70 \%$ ) sensitivity. There is a small but growing literature discussing the efficacy of CTP in the diagnosis of acute posterior ischemia stroke [10-12]. In part, this is the result of limited CTP spatial coverage, illustrated by a recent sub-analysis of the BASICS study, where complete CTP coverage of all posterior fossa ASPECTS regions was only available in
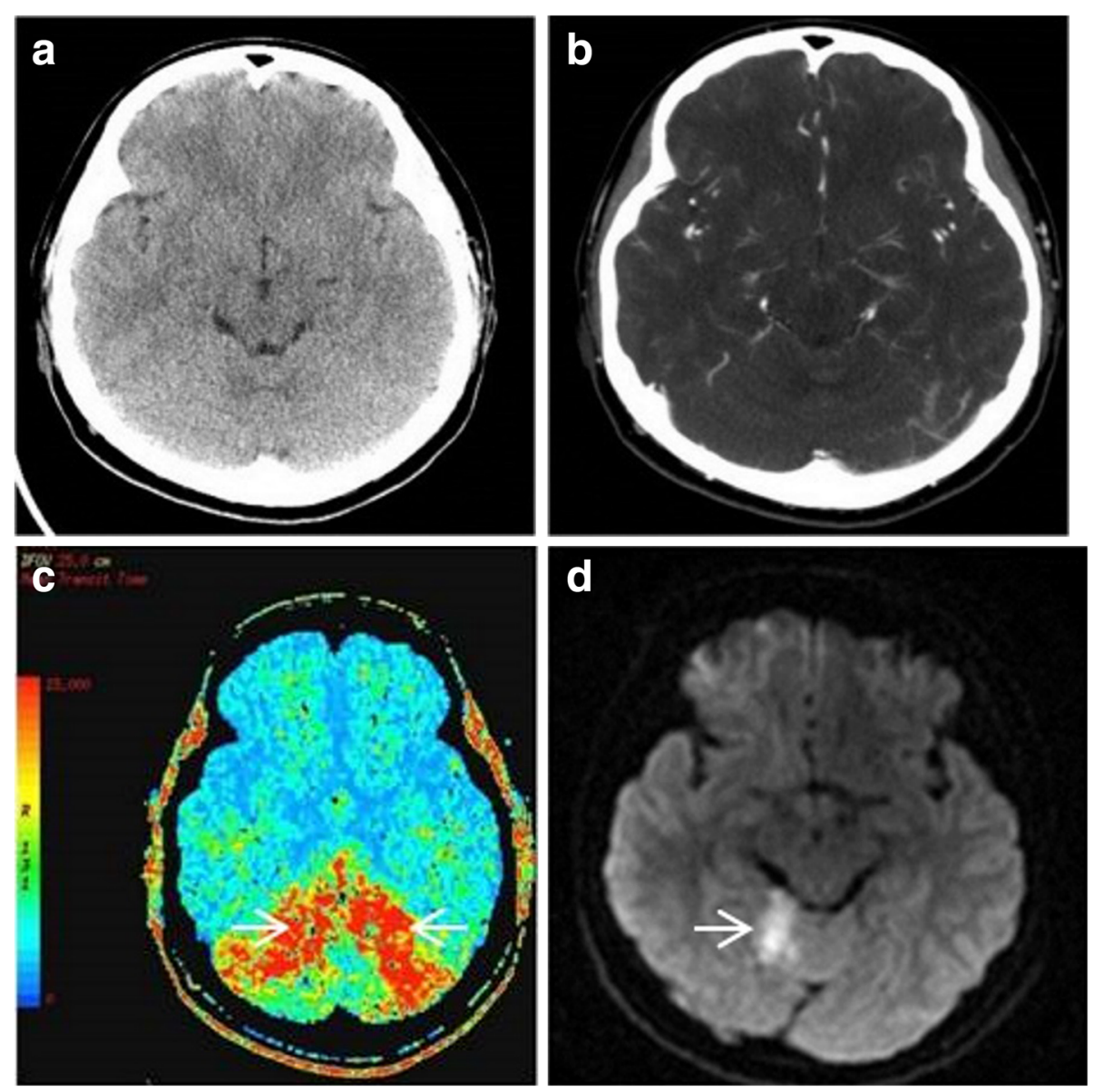

Fig. 2 a NCCT and corresponding (b) CTA-SI, (c) CTP MTT color maps and (d) follow up DWI obtained in 65 year old women presented with posterior ischemic stroke. MTT demonstrates bilateral cerebellar prolongation evolving to right cerebellar hemisphere infarction (arrows). NCCT and CTA-SI are unremarkable 

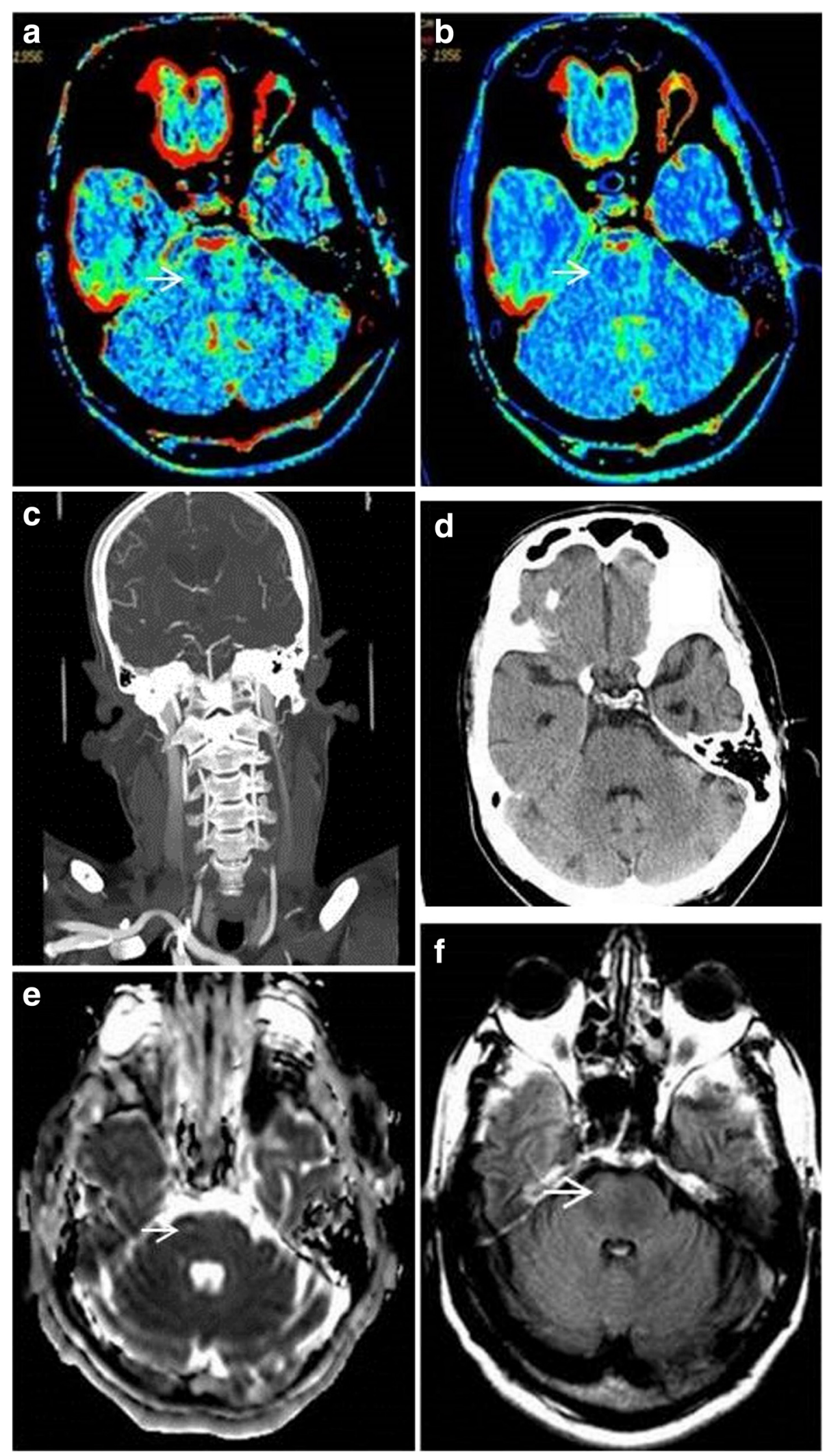

Fig. 3 (See legend on next page.) 
(See figure on previous page.)

Fig. 3 Matched areas of decreased CBF (a) and CBV (b) in the right pons (arrows) indicating an irreversible infraction in a 72 year old man. CTA (c) is negative for arterial filling defects, indicating its lacunar nature. NCCT (d) is equivocal. A right hemipontine acute infarction was confirmed on MRI that was obtained 4 days later as shown on DWI/ADC (e) and corresponding FLAIR (f)

$15 \%$ of 27 patients. While this limitation is addressed by the table-toggle technique $[10,13,23]$ it is associated with reduced sensitivity for detecting small infarcts [23], offset by greater scan coverage contributing to a higher overall detection rate. As 256-multi-detector-CT scanners become increasingly available, whole-brain coverage will become routine $[24,25]$. A prior study, utilizing tabletoggle CTP concluded that the performance of infratentorial CTP is comparable to that of supratentorial CTP [10]. In our study we adopted a pragmatic solution to scanning for centres that do not have a table-toggle option or large array detector scanners. Close collaboration with the stroke team enabled us to alter our traditional supratentorial CTP coverage to the posterior fossa. While stroke triage reflects a typical management pathway in major stroke-centres, targeted posterior fossa CTP is unique and allows simultaneous visualization of lower basal ganglia and posterior fossa assisting in diagnosis of posterior ischemic stroke. This imaging approach represents the application of anatomically and clinically directed scanning, maximizing diagnostic benefit over the risk of radiation exposure. Other important differences between the prior study and this should be noted. Previously, only patients with confirmed infarct on follow-up were investigated whereas we included all patients presenting with acute posterior ischemia stroke irrespective of whether the final diagnosis was TIA or infarction. This approach is supported by the importance of DWI abnormality in the context of clinical TIA [26, 27] and studies demonstrating higher recurrent stroke and TIA risk in TIA patients with initial perfusion abnormality [18]. Further, up to $30 \%$ of patients not receiving rtPA because of mild or rapidly improving ischemic stroke have poor functional outcome $[28,29]$. These findings are supported by the high number $(87 \%)$ of patients included in the present series that demonstrated infarction. The high proportion of patients with posterior ischemic stroke in the current study is attributable to careful clinical screening and is similar to the $86 \%$ prevalence recently reported in a post hoc analysis of posterior fossa infarcts reported by the DUST investigators [11]. To mitigate against a potential performance bias associated with high infarct presence we required precise lateralization for positive infarct designation. Indeed, only $29 \%$ of the vascular territories assessed were affected. This requirement increases the number of false positive and negative designations if overcalling occurred for a particular modality. Prevalence of CTP and CTA-SI abnormality in the context of known posterior fossa infarct in the BASICS sub-study demonstrated MTT, CBF, CBV and CTA-SI changes in 93, 78, 46 and $78 \%$ of patients respectively [12]. These values are higher than 76 and $54 \%$ for any CTP and CTA-SI abnormality reported in this study. The higher prevalence in BASICS is consistent with recruitment of patients with known basilar occlusion rather than any posterior circulatory vessel occlusion included in the present study.

The present cohort size is approximately double that of the prior single center study and included a 12-h compared to 24-h time to presentation. The CT approach therefore reflects the performance of each modality within a screened cohort, assessed within a clinically meaningful time period with greater potential for successful outcome [30].

CTP adds $30 \%$ to the total effective dose of $6.1 \mathrm{mSv}$ for the CTP protocol. Incremental dose, although small, must confer benefit towards diagnosis because even small radiation doses are important in terms of overall population burden. Conscientious effort should therefore be made to conform to the as low as reasonably

Table 4 Distribution of confidence scores for side-specific presence of infarct/ischemia for each modality (levels 1 and 2 indicate definite and probable presence of ischemia/infarction, levels 5 and 6 indicate definite and probable absence of ischemia/infarction)

\begin{tabular}{lllllll}
\hline Modality & \multicolumn{1}{l}{ Distribution of each score, $n(\%)$} & & & \\
\hline Confidence Score & 1 & 2 & 3 & 4 & 5 & 6 \\
NCCT $(n=738)$ & $61(8.3)$ & $47(6.4)$ & $40(5.4)$ & $28(3.8)$ & $244(33.1)$ & $318(43.1)$ \\
CTA-SI $(n=738)$ & $74(10.0)$ & $34(4.6)$ & $14(1.9)$ & $16(2.2)$ & $162(22.0)$ & $438(59.3)$ \\
CTP $(n=738)$ & $133(18.0)$ & $18(2.4)$ & $10(1.4)$ & $20(2.7)$ & $87(11.8)$ & $470(63.7)$ \\
\hline
\end{tabular}

NCCT non-contrast CT, CTA-SI CTA Source Images, CTP CT perfusion 
Table 5 Logistic regression analysis and generalized estimating equations were used to model the real infarct diagnosis on different observed confidence scores for the corresponding modality

\begin{tabular}{|c|c|c|c|c|c|c|}
\hline & \multicolumn{3}{|c|}{ Logistic regression model } & \multicolumn{3}{|l|}{ GEEs method } \\
\hline & $\overline{r^{2}}$ & AlC & $p$-value & OR $(95 \% \mathrm{Cl})$ & QIC & $p$-value \\
\hline \multicolumn{7}{|l|}{ NCCT } \\
\hline Model fit statistics & 0.0856 & 840.6 & & & 840.6 & \\
\hline Observed Confidence Score & & & $<0.0001$ & $0.67(0.61-0.74)$ & & $<0.0001$ \\
\hline \multicolumn{7}{|l|}{$\mathrm{NCCT}+\mathrm{CTA}-\mathrm{SI}$} \\
\hline Model fit statistics & 0.2578 & 686.7 & & & 686.7 & \\
\hline Observed Confidence Score & & & $<0.0001$ & $0.45(0.39-0.51)$ & & $<0.0001$ \\
\hline \multicolumn{7}{|l|}{$N C C T+C T A-S I+C T P$} \\
\hline Model fit statistics & 0.321 & 621 & & & 620.9 & \\
\hline Observed Confidence Score & & & $<0.0001$ & $0.47(0.41-0.52)$ & & $<0.0001$ \\
\hline
\end{tabular}

AIC Akaike information criterion, QIC quasilikelihood information criterion, NCCT non-contrast CT, CTA-SI CTA Source Images, CTP CT perfusion

achievable (ALARA) principle. A recent study shows that no quantitative CTP parameter differences were demonstrated when CTP dose was reduced by $50 \%$ from $100 \mathrm{~mA}$ to $50 \mathrm{~mA}$, however it remains unclear whether performance for infarct detection will remain the same at this reduced dose [31]. Scan obliquity ensures that no significant lens dose exposure occurs unlike CTA which remains the largest single dose contributor. MRI DWI remains the reference standard in acute infarct and provides superior infarct delineation but acute MRI access is limited in many institutions [32]. Therefore, although a recent consensus recommends MRI triage, the decision to treat with IV rtPA is still usually based on NCCT findings [32]. We suggest that CTA and CTP use may improve initial infarct detection over NCCT with the possibility of progressing to MRI where available.

Limitations include the relatively small sample size in comparison to anterior-circulation series. However the present study represents the largest single-center series evaluating pure acute posterior ischemia stroke. In comparison to the DUST study recruiting 88 patients from 14 centers over 4 years we recruited 82 patients in 3 years reflective of a high-volume tertiary institute and arguing against any selection bias. We purposefully utilized multidisciplinary readers of different levels of expertise, reflecting the diversity of everyday practice, where radiology trainees and non-radiologists may be the first to interpret emergency scans before a Neuroradiology staff opinion is sought. The majority of patients received MRI follow-up, with NCCT follow up used only where a radiologically-obvious acute infarct was present. DWI demonstrates high accuracy for stroke detection, but false-negative studies are documented in small and posterior infarcts $[4,33,34]$. It is plausible that a small number of patients may have been misclassified by MRI as negative in the context of a true clinical stroke. Similarly, because any CTP abnormality was considered positive, TIA associated with perfusion deficit, fully recanalized ischemia with residual perfusion defect and intracranial stenosis could all be potentially result in false positive abnormality in the absence of DWI abnormality. The impact of a systematic misclassification error is likely to affect each modality similarly and the effect on modality comparison is presumed negligible.

\section{Conclusions}

In conclusion, in centers with limited CTP coverage, targeted posterior fossa CTP in clinically selected patients enhances confident and correct infarct diagnosis and inter-reader reliability.

Table 6 Inter-reader agreement for three readers as reflected by ICC. ICC of $0.61-0.80$ and $0.81-1.0$ were defined as good and very good concordance, respectively

\begin{tabular}{lllll}
\hline Modality & ICC & $95 \% \mathrm{Cl}$ & Interpretation & $p$-value \\
\hline NCCT & 0.6796 & $0.6034-0.7432$ & Good & reference \\
NCCT + CTA-SI & 0.7829 & $0.7312-0.8260$ & Good & $120.4(<0.0001)$ \\
NCCT + CTA-SI + CTP & 0.8337 & $0.7941-0.8667$ & Very Good & $145.9(<0.0001)$ \\
\hline
\end{tabular}

ICC intraclass-correlation coefficient, NCCT Non-contrast CT, CTA-SI CTA Source Images, CTP CT perfusion 


\section{Competing interests}

The authors declare that they have no competing interests.

\section{Authors' contributions}

MS - data acquisition, CT interpretation, literature research, data analysis and interpretation, manuscript writing. KB - data acquisition, CT interpretation, manuscript revising. RY - CT interpretation, manuscript revising. LZ - performed the statistical analysis and tables, manuscript revising. SS - participated in the design of the study, manuscript revising. MB - data acquisition, manuscript revising. RA - data acquisition, study design, literature research, data analysis and interpretation, manuscript writing. All authors read and approved the final manuscript.

\section{Author details}

${ }^{1}$ Department of Diagnostic Imaging, Division of Neuroradiology, Room AG 31, Sunnybrook Health Sciences Centre, 2075 Bayview Ave, Toronto, ON M4N 3M5, Canada. ${ }^{2}$ Department of Medicine, Division of Neurology, Sunnybrook Health Sciences Centre, 2075 Bayview Ave, Toronto, ON M4N 3M5, Canada.

\section{Received: 27 October 2015 Accepted: 3 January 2016}

\section{Published online: 28 January 2016}

\section{References}

1. Savitz SI, Caplan LR. Vertebrobasilar disease. N Engl J Med. 2005;352:261826.

2. Caplan L. Posterior circulation ischemia: then, now, and tomorrow. The Thomas Willis Lecture. Stroke. 2000;31:2011-23.

3. Hwang DY, Silva GS, Furie KL, Greer DM. Comparative sensitivity of computed tomography vs. magnetic resonance imaging for detecting acute posterior fossa infarct. J Emerg Med. 2012;42:559-65.

4. Oppenheim C, Stanescu R, Dormont D, Crozier S, Marro B, Samson Y, et al. False-negative diffusion-weighted MR findings in acute ischemic stroke. AJNR Am J Neuroradiol. 2000;21:1434-40.

5. Schaefer PW, Yoo AJ, Bell D, Barak ER, Romero JM, Nogueira RG, et al. CT angiography-source image hypoattenuation predicts clinical outcome in posterior circulation strokes treated with intra-arterial therapy. Stroke. 2008 39:3107-9.

6. Koenig M, Klotz E, Luka B, Venderink DJ, Spittler JF, Heuser L, Perfusion CT of the brain: diagnostic approach for early detection of ischemic stroke. Radiology. 1998;209:85-93.

7. Hopyan J, Ciarallo A, Dowlatshahi D, Howard P, John V, Yeung R, et al. Certainty of stroke diagnosis: incremental benefit with $C T$ perfusion over noncontrast CT and CT angiography. Radiology. 2010;255:142-53.

8. Ledezma CJ, Wintermark M. Multimodal CT in stroke imaging: new concepts. Radiol Clin North Am. 2009;47:109-16.

9. Wintermark M, Reichhart M, Cuisenaire O, Maeder P, Thiran JP, Schnyder P et al. Comparison of admission perfusion computed tomography and qualitative diffusion- and perfusion-weighted magnetic resonance imaging in acute stroke patients. Stroke. 2002;33:2025-31.

10. Lee $I H$, You JH, Lee JY, Whang K, Kim MS, Kim YJ, et al. Accuracy of the detection of infratentorial stroke lesions using perfusion $\mathrm{CT}$ : an experimenter-blinded study. Neuroradiology. 2010;52:1095-100.

11. van der Hoeven EJ, Dankbaar JW, Algra A, Vos JA, Niesten JM, van Seeters T, et al. Additional diagnostic value of computed tomography perfusion for detection of acute ischemic stroke in the posterior circulation. Stroke. 2015; 46:1113-5.

12. Pallesen LP, Gerber J, Dzialowski I, van der Hoeven EJ, Michel P, Pfefferkorn T, et al. Diagnostic and Prognostic Impact of pc-ASPECTS Applied to Perfusion CT in the Basilar Artery International Cooperation Study. J Neuroimaging. 2014;25:384-9.

13. Roberts HC, Roberts TP, Smith WS, Lee TJ, Fischbein NJ, Dillon WP, Multisection dynamic CT perfusion for acute cerebral ischemia: the "toggling-table" technique. AJNR Am J Neuroradiol. 2001;22:1077-80.

14. Brenner DJ, Hall EJ. Computed tomography-an increasing source of radiation exposure. N Engl J Med. 2007;357:2277-84.

15. Das T, Settecase F, Boulos M, Huynh T, d'Esterre CD, Symons SP, et al. Multimodal CT provides improved. performance for lacunar infarct detection. AJNR Am J Neuroradiol. 2015;36:1069-75.

16. Puetz V, Sylaja PN, Coutts SB, Hill MD, Dzialowski I, Mueller P, et al. Extent of hypoattenuation on $\mathrm{CT}$ angiography source images predicts functional outcome in patients with basilar artery occlusion. Stroke. 2008;39:2485-90.
17. Sharma M, Fox AJ, Symons S, Jairath A, Aviv RI. CT angiographic source images: flow- or volume-weighted? AJNR Am J Neuroradiol. 2011:32:359-64.

18. Coutts SB, Eliasziw M, Hill MD, Scott JN, Subramaniam S, Buchan AM, et al. An improved scoring system for identifying patients at high early risk of stroke and functional impairment after an acute transient ischemic attack or minor stroke. Int J Stroke. 2008:3:3-10.

19. Asdaghi N, Coutts SB. Imaging predictors of outcome in patients with transient ischemic attacks and minor stroke: review of published data from the VISION study. Eur J Cardiovasc Med. 2011;1:22-5.

20. DeLong ER, DeLong DM, Clarke-Pearson DL. Comparing the areas under two or more correlated receiveroperating characteristic curves: a nonparametric approach. Biometrics. 1988;44:837-45.

21. Zhou XH, Obuchowski NA, McClish DK. The sensitivity and specificity of clustered binary data. In: BaldingDJ, Bloomfield P, Cressie NAC, editors Statistical methods in diagnostic medicine. New York: Wiley; 2002. p. 104-6.

22. Shrout PE, Fleiss JL. Intraclass correlations: uses in assessing rater reliability. Psychol Bull. 1979:86:420-8.

23. Youn SW, Kim JH, Weon YC, Kim SH, Han MK, Bae HJ. Perfusion CT of the brain using 40-mm-wide detector and toggling table technique for initial imaging of acute stroke. AJR Am J Roentgenol. 2008;191:120-6.

24. Mori S, Obata T, Nakajima N, Ichihara N, Endo M. Volumetric perfusion CT using prototype 256-detector row CT scanner: preliminary study with healthy porcine model. AJNR Am J Neuroradiol. 2005;26:2536-41.

25. Murayama K, Katada K, Nakane M, Toyama H, Anno H, Hayakawa M, et al. Whole-brain perfusion CTperformed with a prototype 256-detector row CT system: initial experience. Radiology. 2009;250:202-11.

26. Coutts SB, Hill MD, Simon JE, Sohn CH, Scott JN, Demchuk AM. Silent ischemia in minor stroke and TIApatients identified on mr imaging. Neurology. 2005;65:513-7.

27. Rovira A, Rovira-Gols A, Pedraza S, Grivé E, Molina C, Alvarez-Sabín J. Diffusion-weighted $\mathrm{mr}$ imaging inthe acute phase of transient ischemic attacks. AJNR Am J Neuroradiol. 2002;23:77-83.

28. Rajajee V, Kidwell C, Starkman S, Ovbiagele B, Alger JR, Villablanca P. Early MRI and outcomes of untreated patients with mild or improving ischemic stroke. Neurology. 2006:67:980-4.

29. Barber PA, Zhang J, Demchuk AM, Hill MD, Buchan AM. Why are stroke patients excluded from TPA therapy? An analysis of patient eligibility. Neurology. 2001;56:1015-20.

30. Versnick EJ, Do HM, Albers GW, Tong DC, Marks MP. Mechanical thrombectomy for acute stroke. AJNR Am J Neuroradiol. 2005;26:875-9.

31. Murphy A, So A, Lee TY, Symons S, Jakubovic R, Zhang L, et al. Low dose CT perfusion in acute ischemic stroke. Neuroradiology. 2014;56:1055-62.

32. Wintermark M, Sanelli PC, Albers GW, Bello J, Derdeyn C, Hetts SW, et al. Imaging recommendations for acute stroke and transient ischemic attack patients: A joint statement by the American Society of Neuroradiology, the American College of Radiology, and the Society of Neurolnterventional Surgery. AJNR. 2013;34:117-27.

33. Linfante I, Llinas RH, Schlaug G, Chaves C, Warach S, Caplan LR. Diffusionweighted imaging and National Institutes of Health Stroke Scale in the acute phase of posterior-circulation stroke. Arch Neurol. 2001:58:621-8.

34. Lövblad KO, Laubach HJ, Baird AE, Curtin F, Schlaug G, Edelman RR, et al. Clinical experience with diffusion-weighted MR in patients with acute stroke. AJNR Am J Neuroradiol. 1998;19:1061-6.

\section{Submit your next manuscript to BioMed Central and we will help you at every step:}

- We accept pre-submission inquiries

- Our selector tool helps you to find the most relevant journal

- We provide round the clock customer support

- Convenient online submission

- Thorough peer review

- Inclusion in PubMed and all major indexing services

- Maximum visibility for your research

Submit your manuscript at www.biomedcentral.com/submit
) Biomed Central 